\title{
CHNET-TANDEM experiment: Use of negative muons at RIKEN-RAL Port4 for elemental characterization of
} "Nuragic votive ship" samples

\author{
M. Clemenza, G. Baldazzi, G. Ballerini, M. Bonesini, \\ M. Carpinelli, O. Cremonesi, E. Di Stefano, E. Fiorini,
} G. Gorini, A. Hillier, K. Ishida, A. Menegolli, M. Minoja, E. Mocchiutti, P. Oliva, M. Prata, A. Pullia, M. Rendeli, L.P. Rignanese, M. Rossella, V. Sipala, M. Soldani, L. Tortora, A. Vacchi, E. Vallazza

\section{Published version information}

Citation: M Clemenza et al. "CHNET-TANDEM experiment: use of negative muons at RIKEN-RAL Port4 for elemental characterization of "Nuragic votive ship" samples." Nuclear Instruments and Methods A, vol. 936 (2018): 27-28.

DOI: $10.1016 /$ i.nima.2018.11.076

(C)2018. This manuscript version is made available under the CC-BY-NC-ND 4.0 Licence.

This version is made available in accordance with publisher policies. Please cite only the published version using the reference above. This is the citation assigned by the publisher at the time of issuing the AAM. Please check the publisher's website for any updates. 


\title{
NIMA POST-PROCESS BANNER TO BE REMOVED AFTER FINAL ACCEPTANCE
}

\section{CHNET-TANDEM experiment: Use of Negative Muons at RIKEN-RAL Port4 for elemental characterization of "Nuragic votive ship" samples}

\author{
M. Clemenza ${ }^{\mathrm{a}, \mathrm{b}, *}$, G. Baldazzi ${ }^{\mathrm{c}, \mathrm{d}}$, G. Ballerini ${ }^{\mathrm{a}, \mathrm{e}}$, M. Bonesini $^{\mathrm{a}}$, M. Carpinelli ${ }^{\mathrm{f}, \mathrm{g}}$, O. Cremonesi ${ }^{\mathrm{a}}$, E. Di Stefano ${ }^{\mathrm{a}, \mathrm{s}}$, E. Fiorini $^{\mathrm{a}, \mathrm{b}}$,
} G. Gorini ${ }^{\mathrm{a}, \mathrm{b}}$, A. Hillier ${ }^{\mathrm{h}}$, K. Ishida ${ }^{\mathrm{i}}$, A. Menegolli ${ }^{\mathrm{j}, \mathrm{k}}$, M. Minoja ${ }^{\mathrm{a}, \mathrm{l}}$, E. Mocchiutti ${ }^{\mathrm{m}}$, P. Oliva ${ }^{\mathrm{f}, \mathrm{p}}$, M. Prata ${ }^{\mathrm{k}}$, A. Pullia ${ }^{\mathrm{n}, \mathrm{o}}$, M. Rendeli ${ }^{\mathrm{f}, \mathrm{g}}$, L.P. Rignanese ${ }^{c, d}$, M. Rossella ${ }^{k}$, V. Sipala ${ }^{\mathrm{f}, \mathrm{g}}$, M. Soldani ${ }^{\mathrm{a}, \mathrm{e}}$, L. Tortora ${ }^{\mathrm{q}}$, A. Vacchi $^{\mathrm{m}, \mathrm{r}}$, E. Vallazza $^{\mathrm{m}}$

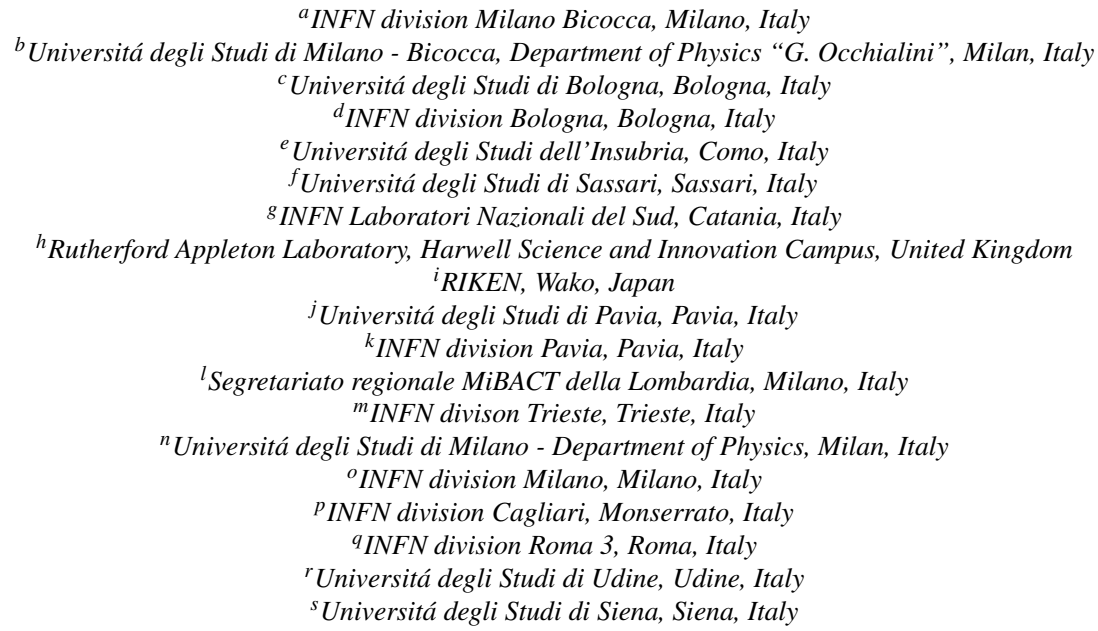

\begin{abstract}
The idea of the CHNET-TANDEM experiment is to develop and optimize a non-destructive technique, which allows analysis deep inside the sample with a good spatial resolution, using a negative muon beam. By selecting the primary muon energies appropriately, bulk analysis can be performed without destructing the sample. The experimental setup used for this experiment, made by 2 hodoscopes and $5 \mathrm{HpGe}$, allowed us to collect very interesting preliminary data concerning scan momentum, positioning and centering of the samples by means of two hodoscopes, analysis of standard material targets and elemental characterization of Nuragic "Bronze Age" votive ship fragments.
\end{abstract}

Keywords: Atomic muon spectroscopy, muonic X-rays, non-destructive bulk analysis PACS: 29.40.Cs, 29.40.Gx

\section{Introduction}

Today, the conservation, preservation and study of cultural heritage is a highly considered field within Italy and Europe. Advanced analytical methods and techniques are an essential

\footnotetext{
${ }^{*}$ Corresponding author

Email address: massimiliano.clemenza@mib.infn.it (M. Clemenza)
} of the material provides key information. The idea is to use the RIKEN-RAL intense pulsed muon beam [1] to develop a nondestructive technique, which allows analysis deep inside the 
material with a good spatial resolution [2]. Because of the large 65 muon mass compared to the electron mass, the muonic X-rays 66 have energies very suitable for standard $\gamma$-ray spectroscopy, so 67 every element is easily recognized. The characteristic muonic 68 X-rays have energies which are about 200 times larger [4] than 69 that of the characteristic X-rays generated for electron beam 70 analysis. Muons also have a high transmission ability and can penetrate much deeper into materials than protons utilized for example in PIXE. Thus, it becomes possible to obtain information about the internal chemical composition of materials up to a few centimeters thick in a non-destructive manner. The goal of the project is to perform, controlling the muon beam momentum, a non-destructive elemental depth-profile analysis [3] of archaeological findings, in materials that can be even several millimeters thick. A proof-of-principle experiment was conducted on the Port 4 beamline in April 2015. During this experiment [5] a new instrument was setup, built and installed into port 4 . We also calibrated the detectors and measured standard samples (Au standards, Bronze Standards and pure elements)[5]. Since then, we have been able to analyze archeological findings, such as Roman and Islamic coins, bronze age artifacts and ancient swords [6, 7].

\section{Experimental set-up}

The experimental setup includes two beam hodoscopes made of scintillating fibers coupled with SiPM, in order to obtain X$\mathrm{Y}$ beam profile information, and $5 \mathrm{HpGe}$-detectors to detect the characteristic X-rays generated from metallic objects irradiated with negative muons (low, medium and high energy muonic X-rays). The aim of the second Hodoscope was to have more precise knowledge regarding the beam focus position and beam direction. This information is crucial for the implementation 71 and validation of the Monte Carlo simulation, which will be need for an accurate quantitative analysis.

\section{Results}

During 4 days of beam time, from 7 th to 11th October 2017, we reached all the objectives expected for the experiment 172028. Also, interesting preliminary results emerged regard- 77 ing the scan beam momentum and the analysis of 4 fragments 78 of Nuragic votive ships [8]. First of all, we were able to opti- 79 mize the position of the sample with respect to the center of the beam, by using scintillating optic fiber sensors read with SiPM $_{80}$ which allow the measurement of the muon flux before and after the sample ??fig1). We also performed a detailed scanning ${ }_{82}^{81}$ (from 28 to $72 \mathrm{MeV} / \mathrm{c}$ with $1 \mathrm{MeV} / \mathrm{c}$ step) of the beam momen- ${ }_{83}$ tum with a multi-layer sample consisting of PTFE, Al, Si, Sn, 84 $\mathrm{Fe}, \mathrm{Cu}, \mathrm{Zn}, \mathrm{Ag}, \mathrm{Ta}, \mathrm{Au}$, layers of variable thickness (250 um ${ }^{85}$ to $1.3 \mathrm{~mm}$ ). The results were compared with a simple model ${ }_{87}^{86}$ for the muon stopping distribution, which highlighted the need ${ }_{88}$ to correct the actual momentum by a factor 1.03 . A more de- 89 tailed quantitative analysis and comparison with the simulations ${ }^{90}$ could provide a better understanding of the correct value of the ${ }_{92}^{91}$ muon momentum and its distribution 2. Finally, we irradiated $49_{9}$ fragments of Nuragic Bronze Age votive ship, from 4 different Sardinian archaeological sites, whose analysis showed interesting differences in the ratio of $\mathrm{Sn} / \mathrm{Cu}$. This gives the possibility of dividing the set of samples into two clusters of common "fabrication provenance", one with ratio $0.10 \pm 0.01$ and one compatible with a ratio of $0.03 \pm 0.01$.

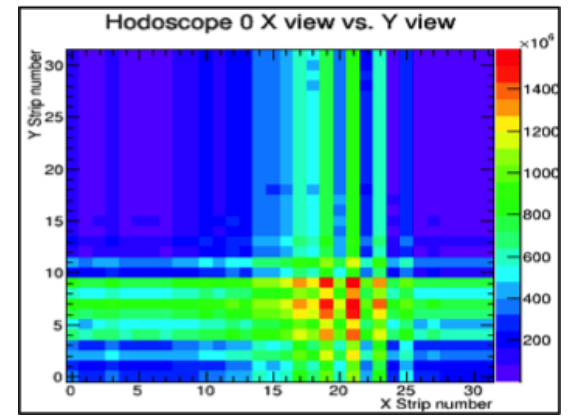

Figure 1: Muon beam intensity acquired by a scintillating optic fiber sensor.

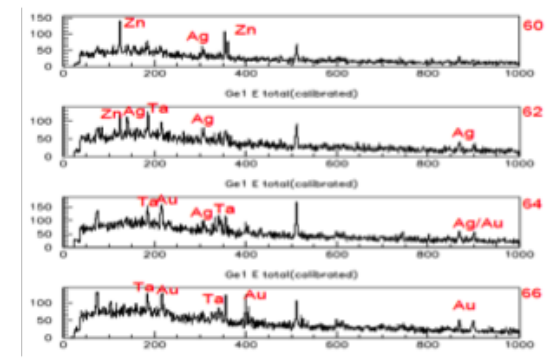

Figure 2: Spectra representing the different momentum of the muon beam.

\section{Conclusions}

The tests carried out showed the possibility of performing non-destructive measurements for the elemental analysis of archaeological samples, which can be characterized both superficially and in depth, by modulating the muon beam momentum.

\section{Acknowledgments}

The Authors would like to thank Gianfranca Salis of the Superintendency of Cagliari for providing the 4 nuragic votive ship samples.

\section{References}

[1] K. Nagamine et al., Hyperfine Interactions. 101-102 (1996) 521-538.

[2] E. Kohler et al,Nucl. Instrum. Meth. 187 (1981) 563-568.

[3] K. Ninomiya et al, Bulletin of the Chemical Society of Japan, (2012) Volume 85228.

[4] K. Nagamine, 2003 Introductory muon science. Cambridge University Press.

[5] A.D. Hillier et al, Microchemical Journal. Vol. 125, (2016), Pages 203-207.

[6] A.D. Hillier,et al in prep, see expt report RB 1520462.

[7] A.I. Wilson 'The metal supply of the Roman Empire', in E. Papi and B. Scardigli (eds), Supplying Rome.

[8] A. Depalmas. Le navicelle di bronzo della Sardegna nuragica. E. Gasperini, 2005 - 394 pagine 